\title{
A Multi-Layered Interdigitative-Electrodes-Based Triboelectric Nanogenerator for Harvesting Hydropower
}

\author{
Zong-Hong Lin ${ }^{\mathrm{a}, \mathrm{b}}$, Gang Cheng ${ }^{\mathrm{a}}$, Xiuhan $\mathrm{Li}^{\mathrm{a}}$, Po-Kang Yang ${ }^{\mathrm{a}}$, Xiaonan Wen ${ }^{\mathrm{a}}$, and \\ Zhong Lin Wang ${ }^{\mathrm{a}, \mathrm{c}, *}$
}

a School of Material Science and Engineering, Georgia Institute of Technology, Atlanta, Georgia 30332-0245, United States

b Institute of Biomedical Engineering, National Tsing Hua University, Hsinchu 30013, Taiwan

c Beijing Institute of Nanoenergy and Nanosystems, Chinese Academy of Sciences, Beijing 100083, China

Corresponding author: School of Material Science and Engineering, Georgia Institute of Technology, Atlanta, Georgia 30332-0245, United States

E-mail: zlwang@gatech.edu 


\begin{abstract}
Hydropower is the most important and wildly-used renewable energy source in the environment. In this paper, we demonstrate a multi-layered triboelectric nanogenerator (TENG) to effectively harvest the water wave energy. For a single-layered TENG, interdigitive electrodes are incorporated in order to generate multiple electric outputs under water wave or water drop impact. For the collection of water wave energy, a polyurethane (PU) coated copper rod is used to roll back and forth and contact with the polytetrafluoroethylene (PTFE) film covered interdigitative electrodes. The surfaces of the PU and PTFE films are fabricated as porous structures and nanowire arrays, which provide an advantages of large contact area and efficient separation. Under one wave impact, the single-layered TENG composed of 9 pairs of interdigitative electrodes can provide 9 pulses of electric outputs (each pulsed output voltage is $52 \mathrm{~V}$ and output current density is $13.8 \mathrm{~mA} \mathrm{~m}^{-2}$ ). The instantaneous output power density of a five-layered TENG is $1.1 \mathrm{~W} \mathrm{~m}^{-2}$. In addition, the PTFE film covered interdigitative electrodes has been successfully used to harvest water drop energy, whcih can also generate 9 pulses of electric outputs upon one water drop falling. All these results show the developed TENG has a potential to harvest the hydropower of ocean wave and raindrop in the near future.
\end{abstract}

Keywords: triboelectric nanogenerator, hydropower, interdigitative electrode, self-powered nanosensor, water wave, water drop 


\section{Introduction}

Over the past decades, climate change has been recognized as the most serious environmental issue affecting our world. Therefore, a great effort has been devoted to discover renewable energy sources with minimized carbon emissions. In addition, less dependence on fossil fuel is mandatory for the sustainable development of the world. In 2012, the first prototype triboelectric nanogenerator (TENG) based on the contact electrification of solid materials was invented to harvest mechanical energy from different sources in the environment, such as wind, ${ }^{[1,2]}$ water, ${ }^{[3]}$ and human motions. ${ }^{[4,5]}$ And four different operation modes have been proposed and demonstrated to show the massive potential of solid-solid TENG, which including vertical contact-separation mode ${ }^{[6,7]}$ lateral sliding mode ${ }^{[8,9]}$ single-electrode mode, ${ }^{[10,11]}$ and freestanding triboelectric-layer mode. ${ }^{[12]}$ Unlimited to energy harvesting application, TENG can further function as self-powered nanosensors by modifying the surfaces of contact materials. The major advantage of self-powered nanosensors is that they can work without external power supply, which have attracted increasing attention in recent years. For examples, self-powered nanosensors based on TENG structure toward catechin molecule, ${ }^{[13]}$ temperature, ${ }^{[14]}$ humidity, ${ }^{[15]}$ metal ion, ${ }^{[16]}$ and light ${ }^{[17]}$ have been widely researched and successfully developed.

Alternatively, scientists also utilized water-solid contact electrification to fabricate a new prototype water-TENG in $2013 .^{[18]}$ The working mechanism of water-TENG is similar to the first prototype TENG based on solid-solid contact electrification, which is a coupling of triboelectric effect and electrostatic induction. The major difference is that in this water-TENG, water itself is one of the contact materials to cause the triboelectric charges and generate electric outputs. For example, the contact electrification between polydimethylsiloxane (PDMS)-patterned pyramid arrays and difference sources of water (deionized, tap, and salt water) has been 
studied and demonstrated with the capability to either collect water wave energy or function as self-powered temperature and ethanol sensors. Recently, another water-TENG was developed to collect the water drop energy based on single-electrode operation mode. ${ }^{[19]}$ This water-TENG successfully showed the potential to harvest the water drop energy under both conditions that the water drop is pre-charged during the traveling process or the triboelectric charges are generated when the water drop is contacted with the superhydrophobic polytetrafluoroethylene (PTFE) film. Scientists have also showed that by changing the superhydrophobic material composition of PTFE to $\mathrm{TiO}_{2}$ nanomaterials, the water-TENG can provide photocatalytic and antibacterial functions when harvesting hydropower. ${ }^{[20]}$ A new active transducer without using any external bias voltage has been sucessfully demonstrated to collect the hydropower from various water motions, ${ }^{[21]}$ which is also based on water-solid contact electrification. These results about water-TENG are important because hydroelectric power is unlimited and could be good alternative to solar energy. ${ }^{[22,23]}$

Different from those previous studies, in this paper we design a multi-layered TENG to effectively harvest the hydropower. For a single-layered TENG, interdigitative electrodes are incorporated in order to generate multiple electric outputs under one water wave or water drop impact. For the collection of water wave energy, a polyurethane (PU) coated copper rod is used to roll back and forth and contact with the PTFE film covered interdigitative electrodes. The working principle is based on solid-solid contact electrification, which is different from that of water-TENG in the previous studies. ${ }^{[18-21]}$ In order to enhance the electric output of TENG, the surfaces of the PU and PTFE films are fabricated as porous structures and nanowire arrays, which provide the advantages of large contact area to generate more triboelectric charges on the surfaces and efficient separation after contact. Under one 
water wave impact, the single-layered TENG composed of 9 pairs of interdigitative electrodes can provide 9 pulses of electric outputs (each pulsed output voltage and

current density can reach $52 \mathrm{~V}$ and $13.8 \mathrm{~mA} \mathrm{~m}^{-2}$, respectively). The instantaneous output power density of a five-layered TENG is $1.1 \mathrm{~W} \mathrm{~m}^{-2}$. The rectified electric outputs have been demonstrated to power light emitting diodes (LEDs). Besides, the part of the PTFE film covered interdigitative electrodes also show the potential to harvest raindrop energy based on water-solid contact electrification, whcih can also generate 9 pulses of electric outputs upon one water drop.

\section{Experimental Section}

\subsection{Preparation of PU and PTFE thin films with nanostructures}

For the polytetrafluoroethylene (PTFE) thin film with nanowire arrays on the surface were synthesized by using a ordered anodic aluminum oxide (AAO) foil as the template and a PTFE solution as the precursor. The AAO template was prepared through a two-step anodization approach. The high-purity aluminum foil (99.99\%) was cleaned, degreased, and annealed at $500{ }^{\circ} \mathrm{C}$ for $3 \mathrm{~h}$. Then the aluminum foil was electropolished in a perchloric acid solution $\left(2.0 \mathrm{M}\right.$ in ethanol) at a $4{ }^{\circ} \mathrm{C}$ for $2 \mathrm{~min} . \mathrm{In}$ the first anodization step, the electropolished aluminum foil was anodized in a oxalic acid solution $(0.3 \mathrm{M})$ at a constant voltage of $40 \mathrm{~V}$ for $40 \mathrm{~h}$. The anodization layer was removed through the wet chemical etching with a solution containing phosphorus acid (0.9 M) and chromium (VI) oxide (0.2 M). Subsequently, a second anodization was carried out under the same condition as the first anodizaion step for $1 \mathrm{~h}$. The AAO template was finally immersed into another phosphoric acid solution to widen the pores. A commercial PTFE precursor was poured into the AAO template and a vacuum process was applied to remove the air remaining in the holes. After curing at ambient temperature for one day, the solvent was evaporated and a PTFE film with 
nanowire arrays on the surface formed. Alternatively, the polyurethane (PU) film surface were fabricated with porous structures. The commercial PU film were etched with a sulfuric acid solution $(3.0 \mathrm{M})$ for 2 min and then cleaned with water.

\subsection{Fabrication of TENG with interdigitative electrodes}

In this part, a poly(methyl methacrylate) (PMMA) mask was curved first by a laser cutter. Then the mask was attached on another PMMA substrate for the deposition of aluminum interdigitative electrodes. Aluminum (thickness around $100 \mathrm{~nm}$ ) was deposited by a RF magnetron sputtering system. The dimensions of the PMMA substrate used in this paper were 3 inch $\times 3$ inch. The width of each aluminum electrode depended on the numbers of total interdigitative electrodes. For example, for the TENG with 9 pairs of interdigitative electrodes, the width of each aluminum electrode was designed to be $0.2 \mathrm{~cm}$. Two conducting wires were connected to the interdigitative electrodes as leads for subsequently electric measurements. The prepared PTFE film was peeled off from the AAO template by using a double-sided tape and directly bonded to the top of interdigitative electrodes. The PU film was also directly attached to a copper rod (diameter about $0.5 \mathrm{~cm}$ ). For the energy harvesting from water drop, we only needed the device of PTFE film covered interdigitative electrodes and operated in single-electrode mode.

\subsection{Characterization}

A Hitachi SU8010 field emission scanning electron microscope (SEM) was used to measure the surface morphologies of PTFE and PU films. For the measurement of electric outputs of TENG, a programmable electrometer (Keithley Model 6514) and a low-noise current preamplifier (Stanford Research System Model SR570) were used. A Linmot linear motor system was applied to provide stable external force making PU 
coated copper rod to move back and forth and contact with the PTFE film covered interdigitative electrodes. This is for the demonstration of single-layered TENG operation. A laboratory platform rocker (260350, Boekel) was used to simulate the the motions of water wave in the environment and enable the multi-layered TENG to work. Finally, a programmable syringe pump (PHD 2000, Harvard Apparatus) was applied to control the volume of each water drop in the experiment of water drop energy harvesting.

\section{Results and Discussion}

The multi-layered TENG can be assembled by numerous single-layered TENGs. Therefore, we use the single-layered TENG as the model to illustrate how the TENG works and what are the components for the basic structure of TENG. The TENG is composed of two parts, one is the PTFE film covered interdigitative electrodes, and the other is a PU coated copper rod (Figure 1a). The PTFE and PU are purposely chosen here because they have a relatively high negative charge affinity and positive charge affinity. ${ }^{[24]}$ In order to increase the electric output of the TENG, both the surfaces of PTFE film and PU are fabricated with nanostructures. The approach for the fabrication of nanostructures is different from other studies, which normally using a plasma reactive ion etching process. ${ }^{[25]}$ The nanowire arrays on PTFE film surface were synthesized by using ordered anodic aluminum oxide (AAO) as the template and a PTFE solution as the precursor. ${ }^{[19,26]}$ The AAO template was prepared by anodizing a $\mathrm{Al}$ foil. ${ }^{[27]}$ The $\mathrm{Al}$ foil was first anodized in $0.3 \mathrm{M}$ oxalic acid solution at a constant voltage of $40 \mathrm{~V}$ for $40 \mathrm{~h}$. The anodization layer was removed through the wet chemical etching with phosphorus acid and chromic acid, and then anodized again under the same condition for $1 \mathrm{~h}$. Afterward, the AAO template was immersed into another phosphoric acid solution to widen the pores. In a typical process, the PTFE 
solution was directly poured into the AAO template and the remaining air inside the nanoholes was removed by using a facile vacuum system. After one day, the solvent evaporated and a PTFE film with nanowire arrays formed (Figure 1b). Finally, the PTFE film was peeled off from the AAO template using a double-sided tape and attached to a poly(methyl methacrylate) (PMMA) substrate, which is deposited with copper interdigitative electrodes. Alternatively, the other contact material of PU are designed to have porous structures. The surface of commercial PU was etched by using diluted sulfuric acid (Figure 1c). ${ }^{[28]}$ Then the PU was covered on a copper rod. The working of the single-layered TENG is based on the rolling of PU coated copper rod over the PTFE film covered interdigitative electrodes (Figure 1d). Under an external force impact, the PU coated copper rod will start to roll and generate the triboelectric charges on the PU and PTFE film surfaces. We will explain the mechanism in detail later. Once the PU coated copper rod roll over 1 pair of interdigitative electrodes, the TENG can generate 1 pulse of electric output. Therefore, the number of electric outputs the TENG can generate will depend on how many pairs of interdigitative electrodes deposited on the PMMA substrate. For example, 9 pulses of electric outputs will be generated if the TENG has 9 pairs of interdigitative electrodes (Figure 3).

The working mechanism of the TENG developed in this paper is based on a coupling effect of triboelectrification and electrostatic induction, which will cause the alternating flow of electrons between the pairs of interdigitative electrodes and contribute to the electric outputs. We define the state I (Figure 2a) and the state II (Figure 2c) as the states when the PU coated copper rod is aligned with electrode A and electrode B, which are a pair of interdigitative electrodes. The intermediate states (Figure $2 \mathrm{~b}$ and Figure 2d) represent the transitional processes in which the PU coated copper rod rolls between the electrode A and the electrode B. Since the PU and PTFE 
are in direct contact, the electrons will transfer from the PU surface to PTFE surface due to the triboelectrification. Therefore, the PU surface will be positively charged and the PTFE surface will be negatively charged. ${ }^{[29]}$ In short-circuit condition, the moving of positively charged object (PU coated copper rod) from state I to state II will cause electrons to flow from the electrode A to the electrode B (Figure 2b) until reaching an equilibrium (Figure 2c). When the PU coated copper rod continues to roll from state II to state I, electrons will flow back from the electrode B to the electrode A (Figure 2d) and reach a new equilibrium (Figure 2a). Therefore, if the TENG has numerous pairs of interdigitative electrodes (Figure S1), multiple electric outputs can be generated when the PU coated copper rod rolls over those PTFE film covered interdigitative electrodes.

To obtain a more quantitative understanding of the proposed working principle, a numerical simulation via finite element method (FEM) has been employed to calculate the potential distribution on the interdigitative electrodes in open-circuit condition. ${ }^{[30]}$ The model using here has the same structure and dimension as the real device. The triboelectric charge density on the PU surface was assigned to be $60 \mu \mathrm{C}$ $\mathrm{m}^{-2}$. Figure 2e-g shows the calculated potential distribution on the interdigitative electrodes during the movement of the PU coated copper rod from the state I to the state II. When the PU coated copper rod is aligned with the electrode (Figure 2e), the state I corresponds to the maximum potential on electrode $\mathrm{A}$ and the minimum potential on electrode $B$, which results in the maximum output open-circuit voltage $\left(V_{\mathrm{oc}}\right)$. Such a voltage then diminishes as the PU coated copper rod starts to move. Once the PU coated copper rod rolls to the middle position (Figure $2 \mathrm{f}$ ), $V_{\text {oc }}$ with the opposite polarity starts to build up until the PU coated copper rod reaches the state II (Figure $2 \mathrm{~g}$ ). Figure $2 \mathrm{~h}$ clearly shows the continuous variation of the $V_{\mathrm{oc}}$ between the state I $(x=0)$ and state II $(x=0.4 \mathrm{~cm})$. Once the TENG has numerous pairs of 
interdigitative electrodes, further rolling of the PU coated copper rod beyond the state II induces the $V_{\text {oc }}$ to change in a reversed way because of the periodic structure. We noticed that the simulation result of $V_{\mathrm{oc}}$ is larger than the measured data (Figure 3a), which is also observed in other studies. ${ }^{[8,30]}$ This could be caused by the setup of boundary condition in FEM analysis. However, it will not affect the relative variation of $V_{\text {oc }}$ on the interdigitative electrodes during the operation of TENG and can help us to understand the working mechanism of TENG. We have also studied the size effect of PU coated copper rod on the TENG output. The simulation result (Figure S2) indicates that the optimized diameter of PU coated copper rod is around $0.5 \mathrm{~cm}$ when fixing both the interdigitative electrode and spacing to be $0.2 \mathrm{~cm}$.

To systematically study the performance of the single-layered TENG, we used a linear motor to control the movements of PU coated copper rod on the PTFE film covered interdigitative electrodes. Figure 3 a shows the output $V_{\text {oc }}$ of a single-layered TENG with 9 pairs of interdigitative electrodes, which can achieve a value of $52 \mathrm{~V}$. The $V_{\mathrm{oc}}$ switches between zero and a maximum value are corresponding to the fully displaced and aligned positions of positively charged PU coated copper rod and interdigitative electrodes, respectively. The results are fully consistent with the mechanism mentioned in Figure 2. An enlarged view of the electric outputs generated from the movement of PU coated copper rod on the PTFE film covered interdigitative electrodes is displayed in Figure 3b, which clearly demonstrates that 9 pulses of electric outputs can be obtained (each pulsed output voltage can reach $52 \mathrm{~V}$ ). The advantage of using interdigitative electrodes here is to introduce a new aproach for more efficient energy harvesting. In contrast to normal TENGs that need the two material surfaces to be fully displaced after contact for a complete transferring of inductive charges, the TENG developed in this paper only requires a displacement of triboelectrically charged object between interdigitative electrodes to generate electric 
outputs, largely improving the energy conversion efficiency. The short-circuit current density $\left(J_{\mathrm{sc}}\right)$ generated by the single-layered TENG also exhibits multiple peaks of alternating directions (Figure 3c). Increasing displacement gives a positive current peak, while decreasing displacement leads to a negative one. No electric current is produced at aligned positions, as illustrated in Figure 2a-d. From the enlarged view of one movement of PU coated copper rod on the PTFE film covered interdigitative electrodes (Figure 3d), 9 pulses of electric currents can also be generated (the average $J_{\mathrm{sc}}$ can reach $13.8 \mathrm{~mA} \mathrm{~m}^{-2}$ ) during the movement.

Next we tried to optimize the energy harvesting efficiency by varying the numbers of interdigitative electrodes on a PMMA substrate with fixed dimensions of $7.5 \mathrm{~cm} \times 7.5 \mathrm{~cm}$. The results are shown in Figure $3 \mathrm{e}$. By integrating the generated output currents of the single layered-TENG with different pairs of interdigitative electrodes, we can deduce the inductively transferred charges on the interdigitative electrodes. It is clearly shown that the single layered-TENG can provide more inductively transferred charges when the interdigitative electrodes are increased from 3 pairs to 9 pairs. However, as the pairs of interdigitative electrodes are further increased from 9 pairs to 13 pairs, the inductively transferred charges start to decrease. This is because that even the single layered-TENG can generate more pulsed electric outputs when increasing the numbers of interdigitative electrodes, it also reduces the electrode width and therefore decreases the quantity of inductively transferred charges. The diameter of the copper rod we use in this paper is about $0.5 \mathrm{~cm}$. We also demonstrated that the moving speed of PU coated copper rod on the PTFE film covered interdigitative electrodes would affect the transferring of inductive charges, which consequently varied the values of output current peaks. In order to observe more clearly, we applied a full-wave diode bridge to rectify the output peaks in the same direction. Figure $3 \mathrm{f}$ indicates that when the moving speed of PU coated copper 
rod changes from $0.2 \mathrm{~m} \mathrm{~s}^{-1}$ to $1.0 \mathrm{~m} \mathrm{~s}^{-1}$, the generated $J_{\mathrm{sc}}$ increases from $5.2 \mathrm{~mA} \mathrm{~m}{ }^{-2}$ to $21.8 \mathrm{~mA} \mathrm{~m}^{-2}$. These results will help to expand the application of single layered-TENG as self-powered sensors, which we will explain in more details later. ${ }^{[31]}$

Another advantage of the TENG is that the single-layered TENG can be easily assembled and become a multi-layered TENG to provide enhanced electric outputs under the same external force impact. The structure of the multi-layered TENG is same as that in Figure $5 \mathrm{c}$ and $5 \mathrm{~d}$. A more clear scheme and device photograph is displayed in Figure S3.

In order to demonstrate this concept, we assembled different numbers of single-layered TENGs in vertical direction and evaluated their performance. A laboratory platform rocker was used to simulate the water wave motion and enable the multi-layered TENG to work. Figure 4a shows the short-circuit current $\left(I_{\mathrm{sc}}\right)$ curves generated by the single-layered TENG, three-layered TENG, and five-layered TENG under the rocker operating at a tilt angle of $10^{\circ}$. It is clearly observed that the electric outputs of TENG can be enhanced by increasing the numbers of single-layered TENG. The average $I_{\mathrm{sc}}$ of five-layered TENG is around $21.5 \mu \mathrm{A}$, which is around 4 times of that generated by the single-layered TENG $(5.3 \mu \mathrm{A})$. We also compared the electric outputs generated by changing the interdigitative electrodes to traditional grating electrodes (Figure S4) and selected copper rod as one of the electric outlets. However, irregular current signals were obtained and the copper wire was easy to detach from the copper rod during the rolling process. These results are not helpful for energy harvesting purposes and development of self-powered sensors. The $I_{\mathrm{sc}}$ curves of single-layered TENG generated by the rocker (Figure 4b) are different from that generated by the linear motor (Figure 3d). This is because when the TENG is driven by the shaker, it causes a gravitational acceleration of the PU coated copper rod to roll over the PTFE film covered interdigitative electrodes. Therefore, the final current 
peak will have a higher but sharper electric signal due to the faster transferring of inductive charges. ${ }^{[32]}$ We further investigated the final output currents of single-layered TENG obtained at different tile angles (Figure 4c). As the tilt angle increases from $2.5^{\circ}$ to $20^{\circ}$, the value of final output current changes from $2.3 \mu \mathrm{A}$ to 8.9 $\mu \mathrm{A}$, which is about 3.9 times. This indicates that the TENG has the potential to be a self-powered sensor which can detect the vibration of ocean wave. To investigate the output power density, we also measured the output voltage and current of the five-layered TENG when connecting to an external load resistor. The resistance was varied from $0.01 \mathrm{M} \Omega$ to $1 \mathrm{G} \Omega$. As the results displayed in Figure $4 \mathrm{~d}$, when the resistance is below $1 \mathrm{M} \Omega$, the output voltage is closed to 0 and the generated current has only little changes. However, when the resistance is varied from $1 \mathrm{M} \Omega$ to $1 \mathrm{G} \Omega$, the output voltage from through the load will generally increase, but the generated current across the load will decrease. As a result, the instantaneous power density generated from the five-layered TENG remain small with the resistance below 0.1 $\mathrm{M} \Omega$ and reach a maximum value of $1.1 \mathrm{~W} \mathrm{~m}^{-2}$ at a resistance of $66 \mathrm{M} \Omega$ (Figure 4e).

To address the potential application of the multi-layered TENG, we further evaluate the electric outputs generated from the five-layered TENG under water wave impact. Polystyrene sheets and an aluminum tube were utilized to support the five-layered TENG on the top of water and ensure the balance of the five-layered TENG after impact. The $V_{\mathrm{oc}}$ of the five-layered TENG is displayed in Figure 5a, which clearly shows multiple outputs can be obtained by each water wave impact. The maximum $V_{\text {oc }}$ can achieve a value of $50 \mathrm{~V}$. The average $I_{\mathrm{sc}}$ generated by the five-layered TENG under water wave impact is $13.5 \mu \mathrm{A}$ (Figure 5b). By using a full-wave diode bridge to rectify the electric outputs in the same direction, commercial LEDs can be lighted up when the PU coated copper rod to roll over the PTFE film covered interdigitative electrodes (Figure 5c, 5d, and Video S1). 
The other importance of developing interdigitative electrodes-based TENG is that it can enhance the harvesting efficiency of water drop energy. To address the potential of this concept, we used a commercial fluorinated ethylene propylene (FEP) film to replace the role of nanostructured PTFE film in the as-developed TENG. The reason we choose FEP film is because its surface is hydrophobic. From previous studies, we have learned that the necessary of using hydrophobic film in water-TENG which is based on solid-water contact electrification. ${ }^{[19,20]}$ To measure the performance of the water-TENG (FEP film covered interdigitative electrodes), we used a programmable syringe pump to control the volume of each water drop. The volume of each water drop was $30 \mu \mathrm{L}$ and fell from a height of $20 \mathrm{~cm}$. The scheme to represent how the water drop interact with the water-TENG is shown in Figure S5. The water drop would contact with the FEP film and pass throuth the water-TENG. To highlight future application, untreated tap water was selected as the water source. Figure 5e shows the output currents of water-TENG under the impact of continuous water drops. The output current reaches an average value of $0.3 \mu \mathrm{A}$ and exhibits $\mathrm{AC}$ behavior, with an equal number of electrons flowing in the opposite direction in each cycle. The experimental data validates the working mechanism proposed in our previous study. ${ }^{[19]}$ An enlarged view of the output currents contributed from one water drop is displayed in Figure 5f. 9 pulses of output currents can be provided by the water-TENG. Under the same condition, normal water-TENG can only generate one pulse of electric output under one water drop impact. It is observed that the current peak corresponding to the first pair of interdigitative electrodes has a higher magnitude but lasts shorter than that generated by other pairs of interdigitative electrodes. This can be explained by the fact that the falling water drop has a higher speed when passing through the first pair of interdigitative electrodes and thus generate a larger but narrower current signal. The other reason we select FEP film to 
demonstrate is because of its transparency. By changing the PMMA substrate and Al electrodes to PET film and indium tin oxide (ITO) electrodes, the water-TENG can become a highly transparent one. Figure S6 shows the transmittance spectra of ITO interdigitative electrodes-deposited PET film before and after FEP film covering. By combining the highly transparent water-TENG with solar cell, a new hybrid energy cell which can simultaneously or individually harvest solar and raindrop energy in different weather conditions. ${ }^{[33]}$

\section{Conclusion}

In summary, a multi-layered TENG with interdigitative electrodes has been successfully developed to harvest the hydropower in this paper. For a single-layered TENG with 9 pairs of interdigitative electrodes, 9 pulses of electric outputs $\left(V_{\text {oc }}=52\right.$ $\mathrm{V}, J_{\mathrm{sc}}=13.8 \mathrm{~mA} \mathrm{~m}^{-2}$ ) are generated in one time upon the operation of TENG. Different from the previous TENG designs, using a free rolling contact material to construct the TENG shows the potential to detect the vibration of water wave without any electricity input, whcih is a concept of self-powered sensor. By assembling five single-layered TENGs to become one, it provides an output power density of $1.1 \mathrm{~W}$ $\mathrm{m}^{-2}$ under one water wave impact. Not limited to the numbers of interdigitative electrodes and dimensions of TENG we demonstrate in this paper, the novelty of this concept is that the multi-layered TENG can be easily to expand for the future applications.

\section{Supporting Information}

Electronic Supporting Information (ESI) is available online.

\section{Acknowledgements}


This work was supported by Office of Basic Energy Sciences (DE-FG02-07ER46394), U.S. Department of Energy, NSF, Taiwan Ministry of Science and Technology (103-2113-M-007-021-MY2), and the "thousands talents" program for pioneer researcher and his innovation team, China, Beijing City Committee of science and technology project (Z131100006013004 and Z131100006013005). Patents have been filed based on the research presented here.

\section{References}

[1] Y. Xie, S. Wang, L. Lin, Q. Jing, Z.-H. Lin, S. Niu, Z. Wu, Z. L. Wang, $A C S$ Nano 2013, 7, 7119.

[2] Y. Yang, G. Zhu, H. Zhang, J. Chen, X. Zhong, Z.-H. Lin, Y. Su, P. Bai, X. Wen, Z. L. Wang, ACS Nano 2013, 7, 9461.

[3] Y. Hu, J. Yang, Q. Jing, S. Niu, W. Wu, Z. L. Wang, ACS Nano 2013, 7, 10424.

[4] P. Bai, G. Zhu, Z.-H. Lin, Q. Jing, J. Chen, G. Zhang, J. Ma, Z. L. Wang, ACS Nano 2013, 7, 3713.

[5] G. Zhu, Z.-H. Lin, Q. Jing, P. Bai, C. F. Pan, Y. Yang, Y. S. Zhou, Z. L. Wang, Nano Lett. 2013, 13, 847.

[6] F.-R. Fan, Z.-Q. Tian, Z. L. Wang, Nano Energy 2012, 1, 328.

[7] F.-R. Fan, L. Lin, G. Zhu, W. Wu, R. Zhang, Z. L. Wang, Nano Lett. 2012, 12, 3109.

[8] S. Wang, L. Lin, Y. Xie, Q. Jing, S. Niu, Z. L. Wang, Nano Lett. 2013, 13, 2226.

[9] G. Zhu, J. Chen, Y. Liu, P. Bai, Y. S. Zhou, Q. Jing, C. F. Pan, Z. L. Wang, Nano Lett. 2013, 13, 2282.

[10] Y. Yang, Y. S. Zhou,; H. Zhang, J. Chen, Y. Liu, S. Lee, Z. L. Wang, Adv. Mater. 2013, 25, 6594 .

[12] G. Cheng, Z.-H. Lin, Z. Du, Z. L. Wang, Adv. Funct. Mater. 2014, 24, 2892. 
[12] S. Wang, Y. Xie, S. Niu, L. Lin, Z. L. Wang, Adv. Mater. 2014, 26, 2818.

[13] Z.-H. Lin, Y. Xie, Y.Yang, S. Wang, G. Zhu, Z. L. Wang, ACS Nano 2013, 7, 4554

[14] X. Wen, Y. Su, Y. Yang, H. Zhang, Z. L. Wang, Nano Energy. 2014, 4, 150.

[15] V. Nguyen, R. Yang, Nano Energy. 2014, 2, 604.

[16] Z.-H. Lin, G. Zhu, Y. S. Zhou, Y. Yang, P. Bai, J. Chen, Z. L. Wang, Angew. Chem. Int. Ed. 2013, 52, 5065.

[17] Z.-H. Lin, G. Cheng, Y. Yang, Y. S. Zhou, S. Lee, Z. L. Wang, Adv. Funct. Mater. 2014, 24, 2810.

[18]Z.-H. Lin, G. Cheng, L. Lin, S. Lee, Z. L. Wang, Angew. Chem. Int. Ed. 2013, 52, 12545.

[19] Z.-H. Lin, G. Cheng, S. Lee, K. C. Pradel, Z. L. Wang, Adv. Mater. 2014, 26, 4690.

[20] Z.-H. Lin, G. Cheng, W. Wu, K. C. Pradel, Z. L. Wang, ACS Nano 2014, 8, 6440.

[21] S.-H. Kwon, J. Park, W. K. Kim, Y. J. Yang, E. Lee, C. J. Han, S. Y. Park, J. Lee, Y. S. Kim, Energy. Environ. Sci. 2014, 7, 3279.

[22] M. Ma, L. Guo, D. G. Anderson, R. Langer, Science 2013, 339, 186.

[23] G. Cheng, Z.-H. Lin, Z. Du, Z. L. Wang, ACS Nano 2014, 8, 1932.

[24] L. S. McCarty, G. M. Whitesides, Angew. Chem. Int. Ed. 2008, 47, 2188.

[25] G. Zhu, Y. Su, P. Bai, J. Chen, Q. Jing, W. Yang, Z. L. Wang, ACS Nano 2014, 8, 6031.

[26] S. Lee , J. H. Kang, S. J. Lee , W. Hwang, Lab Chip 2009, 9, 2234

[27] H. Masuda, K. Fukuda, Science 1995, 268, 1466.

[28] M. Suzuki, T. Takahashi, S. Aoyagi, Micromachines 2012, 3, 315.

[29] F. Galembeck, T. A. L. Burgo, L. B. S. Balestrin, R. F. Gouveia, C. A. Silva, A. Galembeck, RSC Adv. 2014, 4, 64280. 
[30] G. Cheng, L. Zheng, Z.-H. Lin, J. Yang, Z. Du, Z. L. Wang, Adv. Energy Mater. 2015, Early View (DOI: 10.1002/aenm.201401452).

[31] S. Wang, L. Lin, Z. L. Wang, Nano Lett. 2012, 12, 6339.

[32] Y. Yi, L. Lin, S. Niu, J. Yang, W. Wu, S. Wang, Q. Liao, Y. Zhang, Z. L. Wang, Adv. Funct. Mater. 2014, 24, 7488.

[33] L. Zheng, Z.-H. Lin, G. Cheng, W. Wu, X. Wen, S. Lee, Z. L. Wang, Nano Energy 2014, 9, 291.

\section{Figure Captions}

Figure 1. (a) Schematic diagram of a typical single-layered TENG. The TENG contains a PTFE film covered interdigitative electrodes and PU coated copper rod. The surfaces of PTFE film and PU are fabricated with nanostructures. (b, c) SEM images of the PTFE film and PU surfaces. The morphologies of PTFE film and PU are nanowire arrays and porous structures. (d) Illustration of TENG working principle. The operation of TENG is based on the movement of PU coated copper rod on the PTFE film covered interdigitative electrodes.

Figure 2. (a-d) Working mechanism of the interdigitative electrodes-based TENG. The rolling of PU coated $\mathrm{Cu}$ rod on PTFE film causes electrons to transfer from PU surface to PTFE film surface. (a) At the state I, the positively charged PU coated $\mathrm{Cu}$ rod is on the top of electrode A, and therefore electrons will accumulate at electrode A. (b) Once the PU coated $\mathrm{Cu}$ rod starts to roll, it creates a positive electric potential and electrons flow from electrode A to electrode B to balance the potential difference, contributing to a positive output current. (c) At the state II, the positively charged PU coated $\mathrm{Cu}$ rod is on the top of electrode $\mathrm{B}$, and therefore electrons accumulate at electrode B. (d) The PU coated Cu rod starts to move forward to the next electrode A 
creates a negative electric potential, and electrons will flow from electrode B back to electrode A. This generates a negative output current. Once the PU coated $\mathrm{Cu}$ rod rolls back and forth on the PTFE film covered interdigitative electrodes, electric outputs will be continuously generated. The numbers of electric outputs depend on the pairs of interdigitative electrodes. (e-g) Calculated potential distribution on the interdigitative electrodes via finite element method. The model using here has the same structure and dimension as the real device. The triboelectric charge density on the PU surface is assigned to be $60 \mu \mathrm{C} \mathrm{m}^{-2}$. (h) Continuous variation of the $V_{\mathrm{oc}}$ between the state I $(x=0)$ and state II $(x=0.4 \mathrm{~cm})$.

Figure 3. (a) Output $V_{\mathrm{oc}}$ and (c) output $J_{\mathrm{sc}}$ of a single-layered TENG with 9 pairs of interdigitative electrodes generated by using a linear motor system to control the movement of PU coated copper rod. (b) and (d) are the enlarged electric output curves of (a) and (c), respectively. (e) Impact of the numbers of interdigitative electrodes on the inductively transferred charges of a single-layered TENG. The PMMA substrate dimensions are confined as $7.5 \mathrm{~cm} \times 7.5 \mathrm{~cm}$. (f) Dependence of the rectified $J_{\mathrm{sc}}$ of a single-layered TENG on the moving speed of PU coated copper rod.

Figure 4. (a) Output $I_{\mathrm{sc}}$ of a single-layered TENG, three-layered TENG, and five-layered TENG generated by a laboratory platform rocker swinging at a tilt angle of $10^{\circ}$. (b) Enlarged view of one cycle of the output $I_{\mathrm{sc}}$ from a single-layered TENG in (a). (c) Impact of the $8_{\text {th }}$ output $I_{\text {sc }}$ peak value from a single-layered TENG on different rocker tilt angles. (d, e) Dependence of output voltage (d), output current (d), and output power density (e) of the five layered-TENG on the resistance of external load. 
Figure 5. (a) Output $V_{\mathrm{oc}}$ and (b) output $I_{\mathrm{sc}}$ of the five-layered TENG generated by the vibration of water waves. (c) Photograph of the five-layered TENG integrated with polystyrene sheets and 16 LEDs. (d) Photograph of 16 LEDs lightened up by the rectified electric outputs from the five-layered TENG. (e) Output $I_{\mathrm{sc}}$ of the PTFE film covered interdigitative electrodes generated by water drops. (f) Enlarged view of Output $I_{\text {sc }}$ generated by water drops in (e). (f) clearly shows that multiple electric outputs can be generated by a single water drop. The volume of each water drop is fixed at $30 \mu \mathrm{L}$ and falls from a height of $20 \mathrm{~cm}$.

\section{Figures}

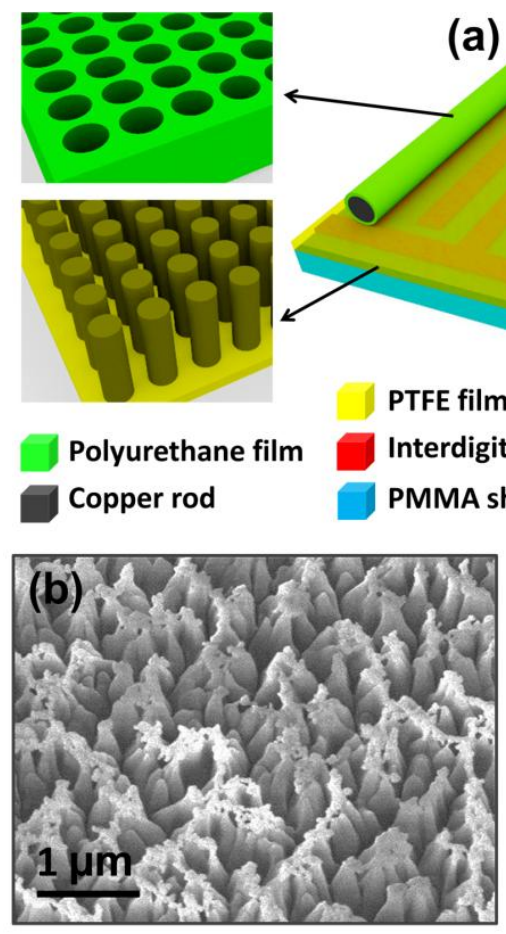

(a)
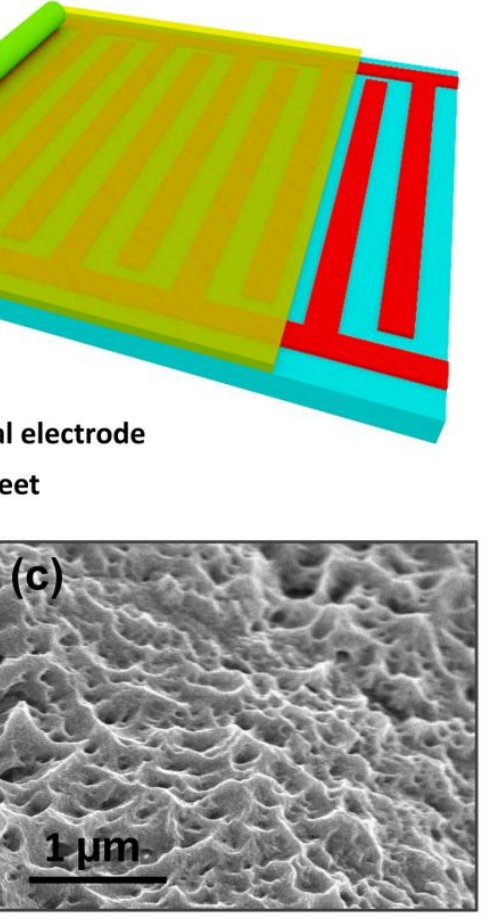

(d)

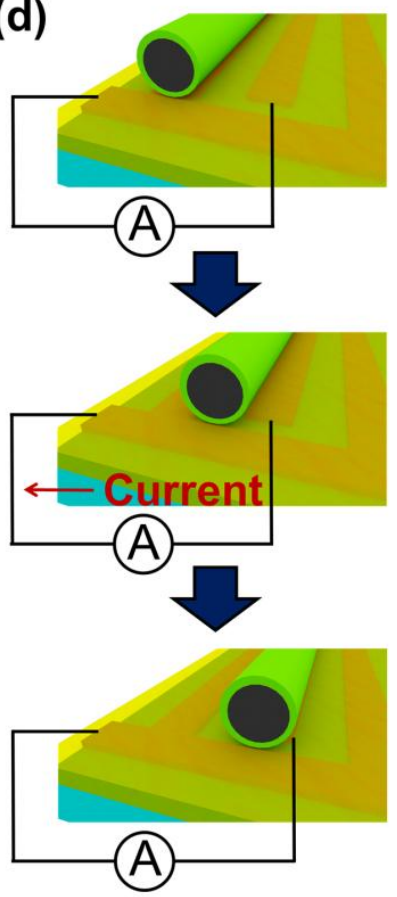

Figure 1 
(a)

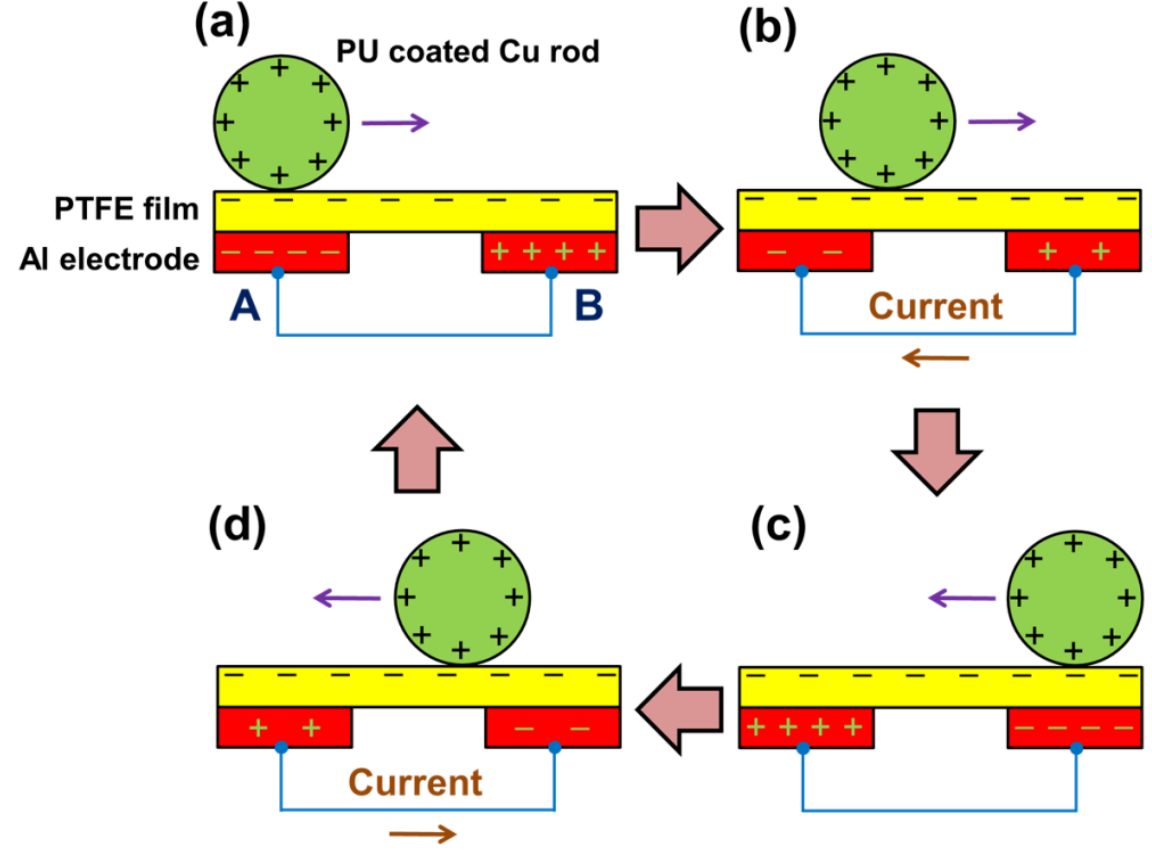

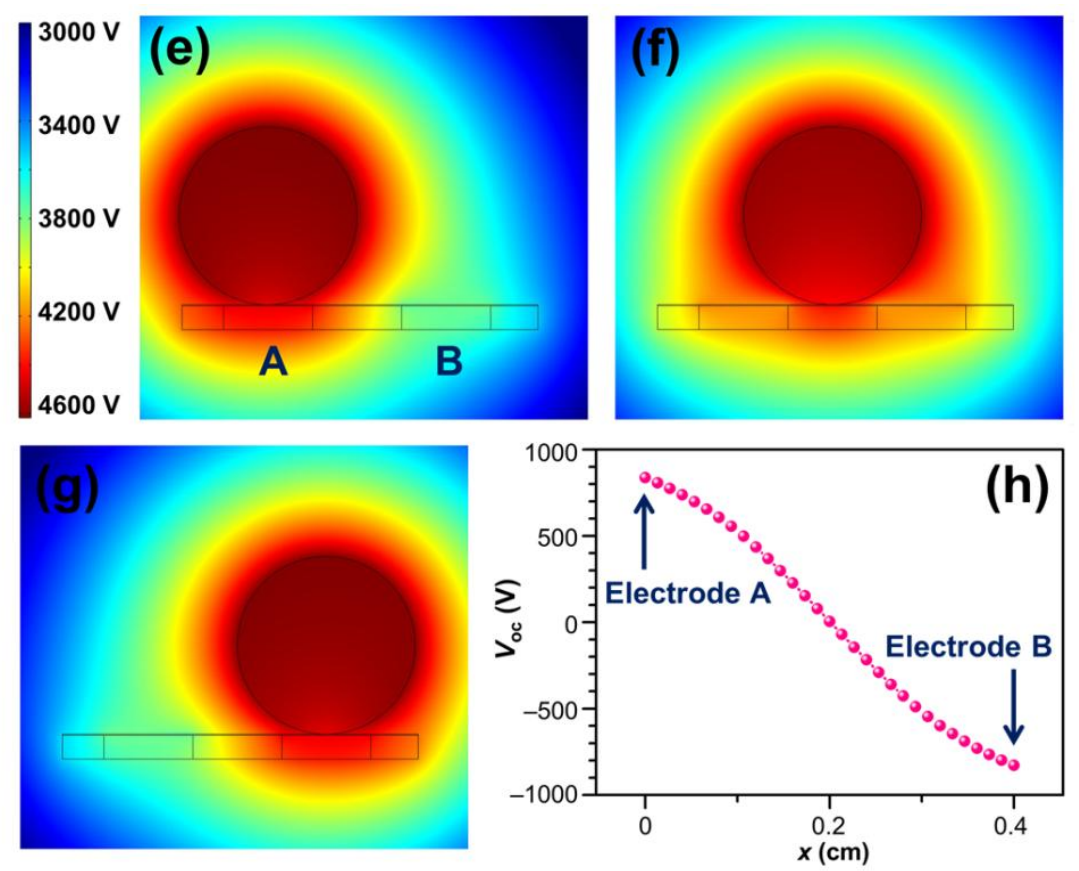

Figure 2 

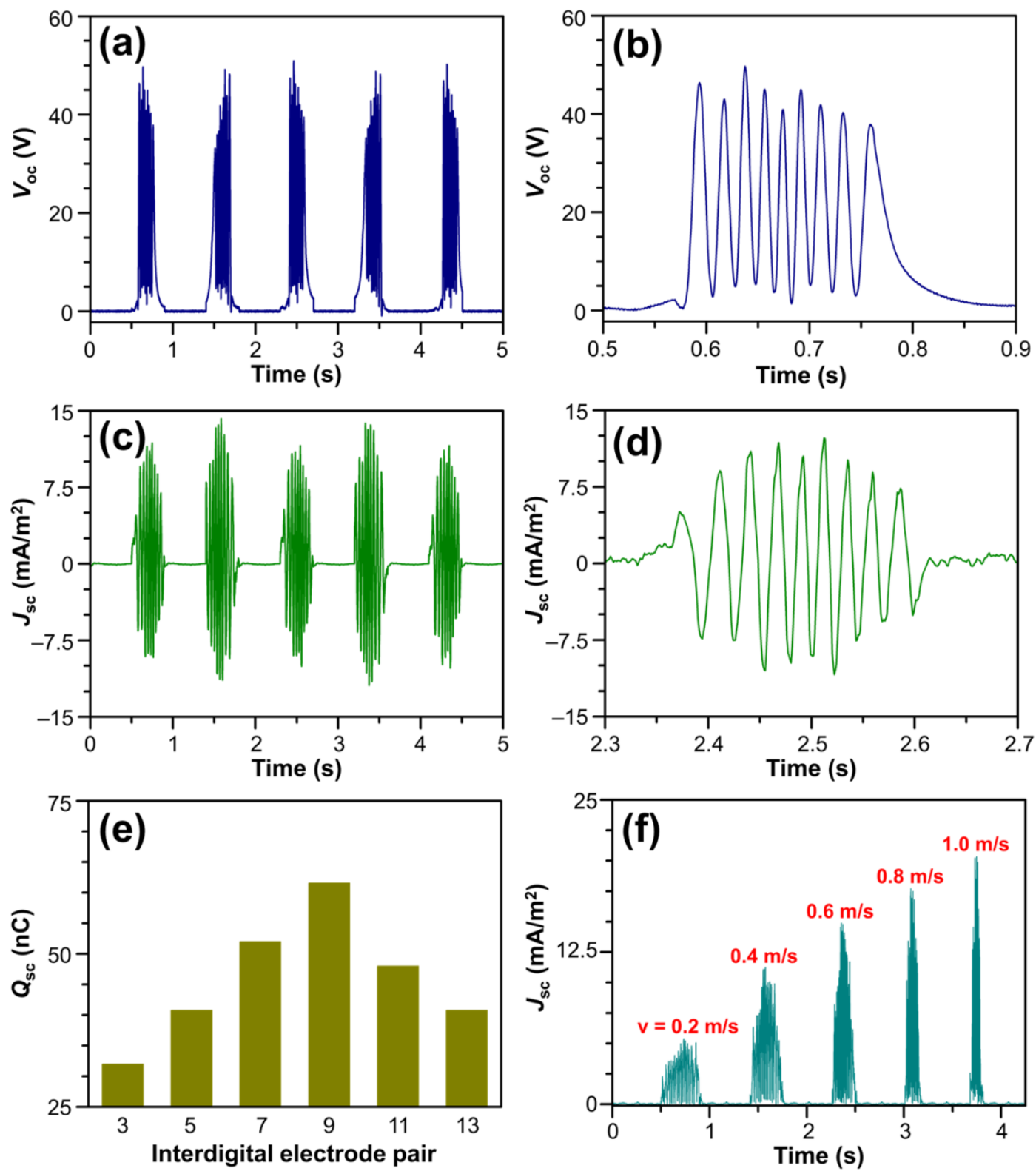

Figure 3 

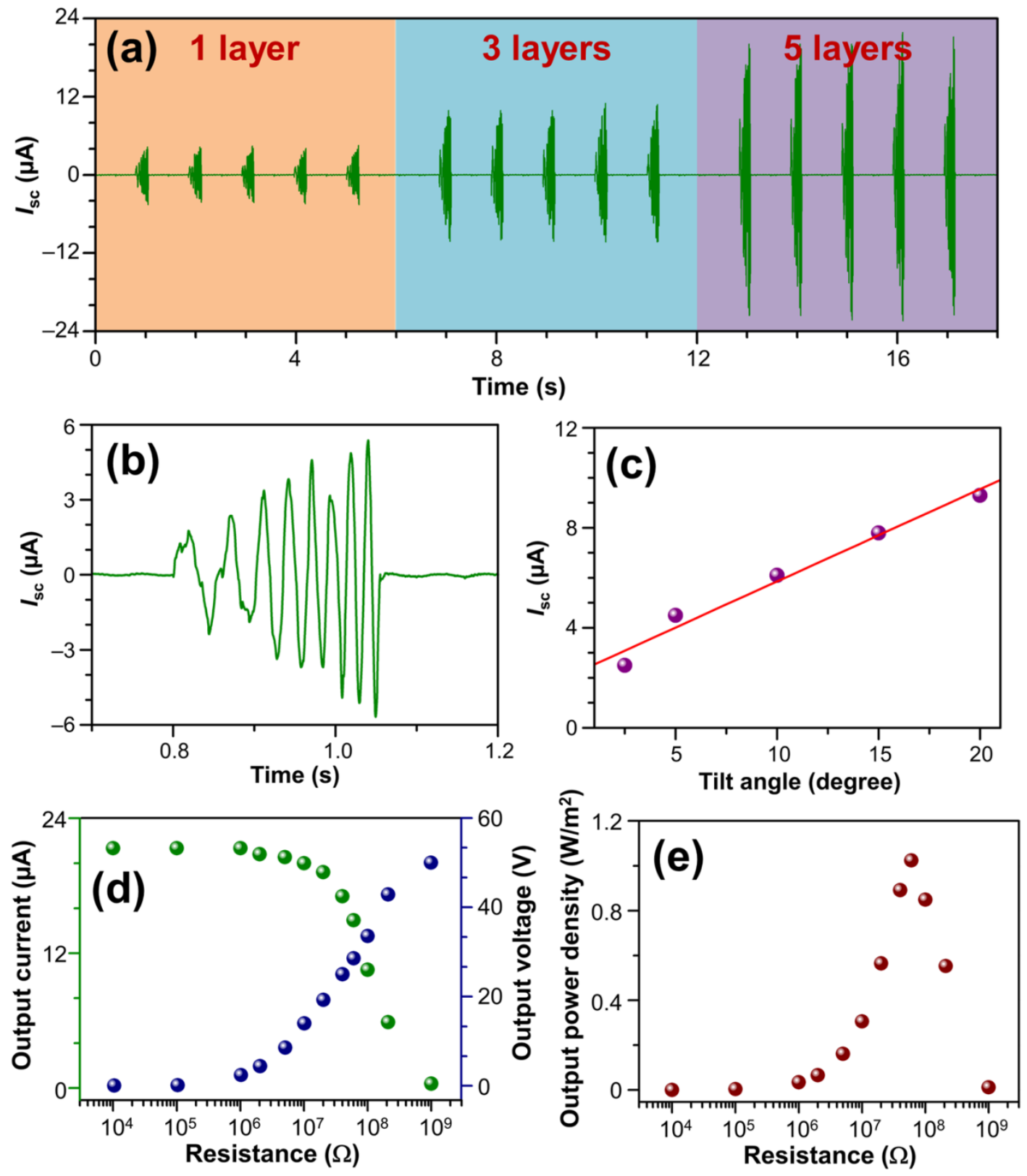

Figure 4 

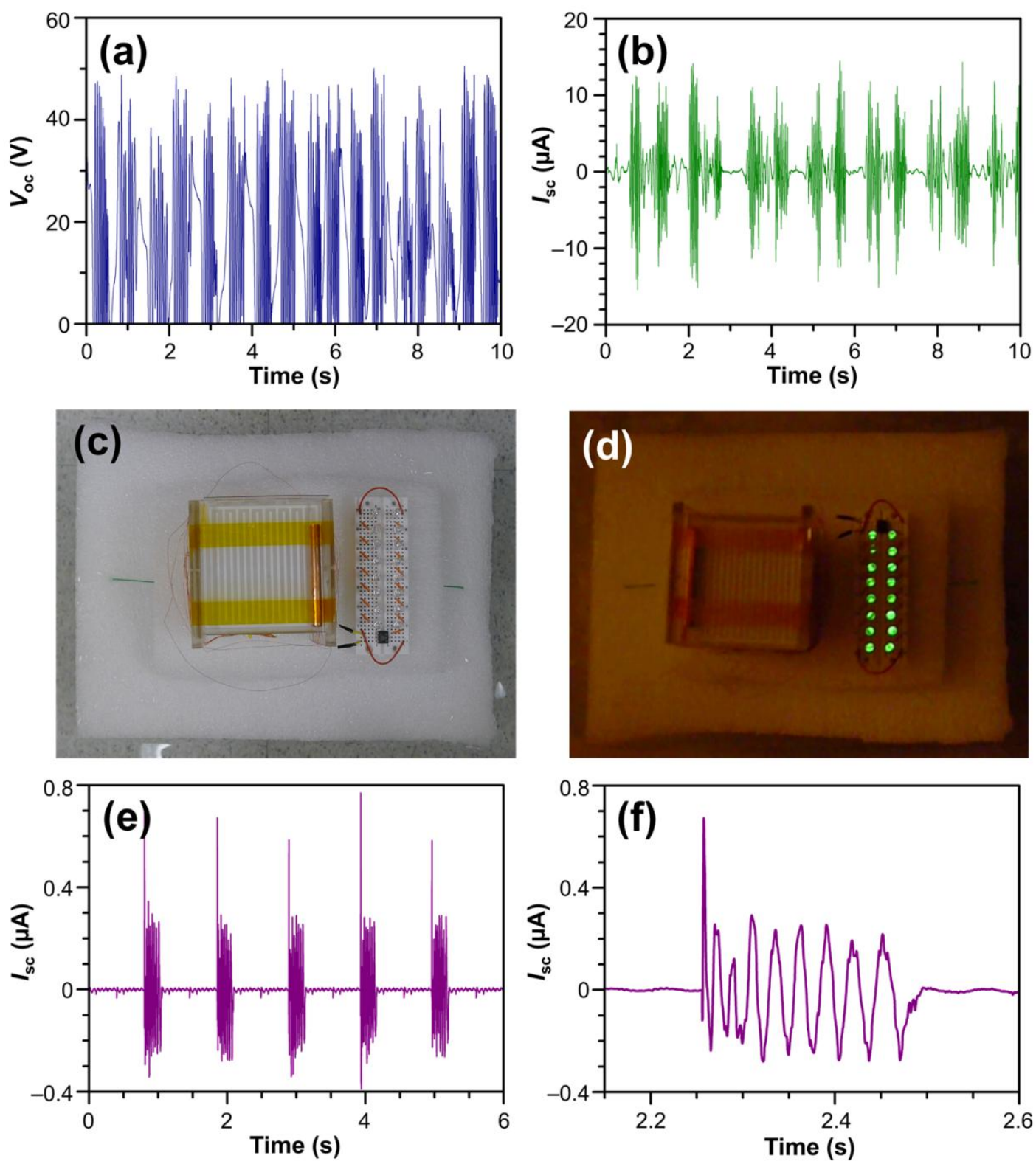

Figure 5 


\section{Author Biography}

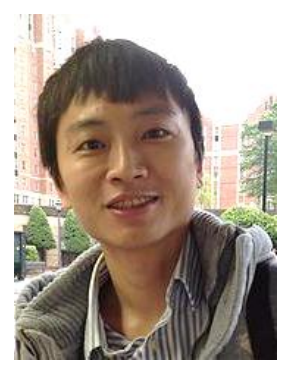

Zong-Hong Lin received his Ph.D degree from Department of Chemistry, National Taiwan Univerdity in 2009. Before joining the Institute of Biomedical Engineering, National Tsing Hua University in Taiwan as an assistant professor. He was a postdoctoral researcher supervised by Professor Zhong Lin Wang in the school of Materials Science and Engineering at Georgia Institute of Technology. His research interests include the development of high-output generators with novel design for efficient energy harvesting, self-powered systems for biomolecules detection and environmental sensors, highely efficient and stable catalysts for fuel cell applications, and fabrication of metal miconductor nanowires

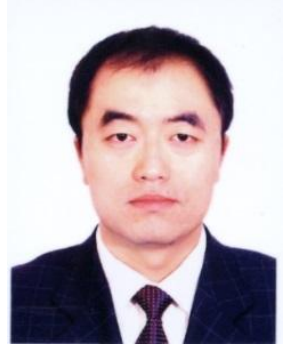

Gang Cheng is visiting scholar in School of Materials Science and Engineering at Georgia Institute of Technology and an associate professor in Henan University. He received the B.S. degree from Henan University in 2000 and the Ph.D. degree from Jilin University in 2008. His research interests are nanostructure-based electronic and optoelectronic devices, nanogenerator, and self-powered nanosensors.

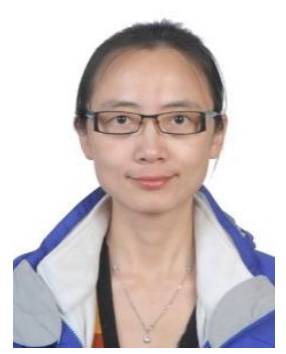

Xiuhan Li received the B.S. degree (2000) and the M.S. degree(2003) in Microelectronics from Changchun Institute of Optics and Fine Mechanics, Changchun. She received her Ph.D. degree (2006) from Peking University, Beijing, China. Then, she worked as a lecturer, associate professor (2010) in the school of Electronics and Information Engineering in Beijing Jiaotong University. In 2014 she worked as visiting scholar in the group of Professor Zhong Lin Wang at Georgia Institute of Technology. Her main research interest and activities are: RF MEMS devices for wireless communication and energy transfer system, self-powered nano/micro-system, and MEMS devices for $\mathrm{THz}$ communication system. 


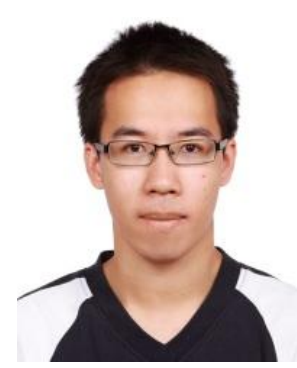

Po-Kang Yangreceived the B.S. degree in material science from the National Chung HsingUniversity, Taichung, Taiwan, in 2006 and the M.S.degree in material science and engineering fromthe National Taiwan University, Taipei, Taiwan, in2008, where he is currently working toward thePh.D. degree in optoelectronic engineering,National Taiwan University, Taipei, Taiwan. During hisM.S. research, he centered on fabricating anddesigning a photodetector.His major research focuses on the developmentof resistive random access memory and triboelectric nanogenerator.

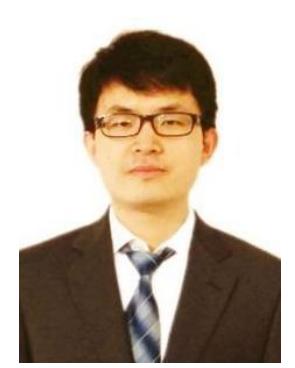

Xiaonan Wen received his B.S. degree in Physics from Peking University, China in 2010. He is currently a Ph.D. student in the School of Materials Science \& Engineering, Georgia Institute of Technology under the supervision of Prof. Zhong Lin Wang. Xiaonan's research interests include synthesis of functional nanomaterials; energy harvesting using piezoelectric and triboelectric generators; self-powered nanosystems; piezo-electronics and piezo-optoelectronics based on $\mathrm{ZnO}, \mathrm{GaN}$ nanowires/thin films for novel transistors, strain sensors, chemical sensors and optoelectronic devices; flexible electronics based on advanced polymer and glass substrates; integration of novel devices into functional systems.

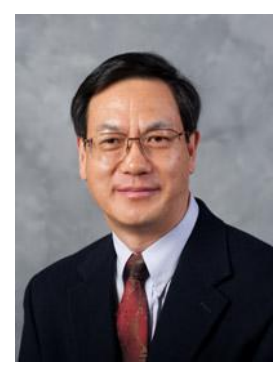

Zhong Lin Wang received his Ph.D. from Arizona State University in physics. He is now the Hightower Chair in Materials Science and Engineering, Regents' Professor, Engineering Distinguished Professor and Director, Center for Nanostructure Characterization, at Georgia Tech. Dr. Wang has made original and innovative contributions to the synthesis, discovery, characterization and understanding of fundamental physical properties of oxide nanobelts and nanowires, as well as applications of nanowires in energy sciences, electronics, optoelectronics and biological science. His discovery and breakthroughs in developing nanogenerators established the principle and technological roadmap for harvesting mechanical energy from the environment and biological systems for powering a personal electronics. His research on self-powered nanosystems has inspired the worldwide effort in academia and industry for studying energy for micro-nano-systems, which is now a distinct disciplinary in energy research and 
future sensor networks. He coined and pioneered the field of piezotronics and piezo-phototronics by introducing piezoelectric potential gated charge transport process in fabricating new electronic and optoelectronic devices. Details can be found at: http://www.nanoscience.gatech.edu. 


\section{Graphical Abstract}
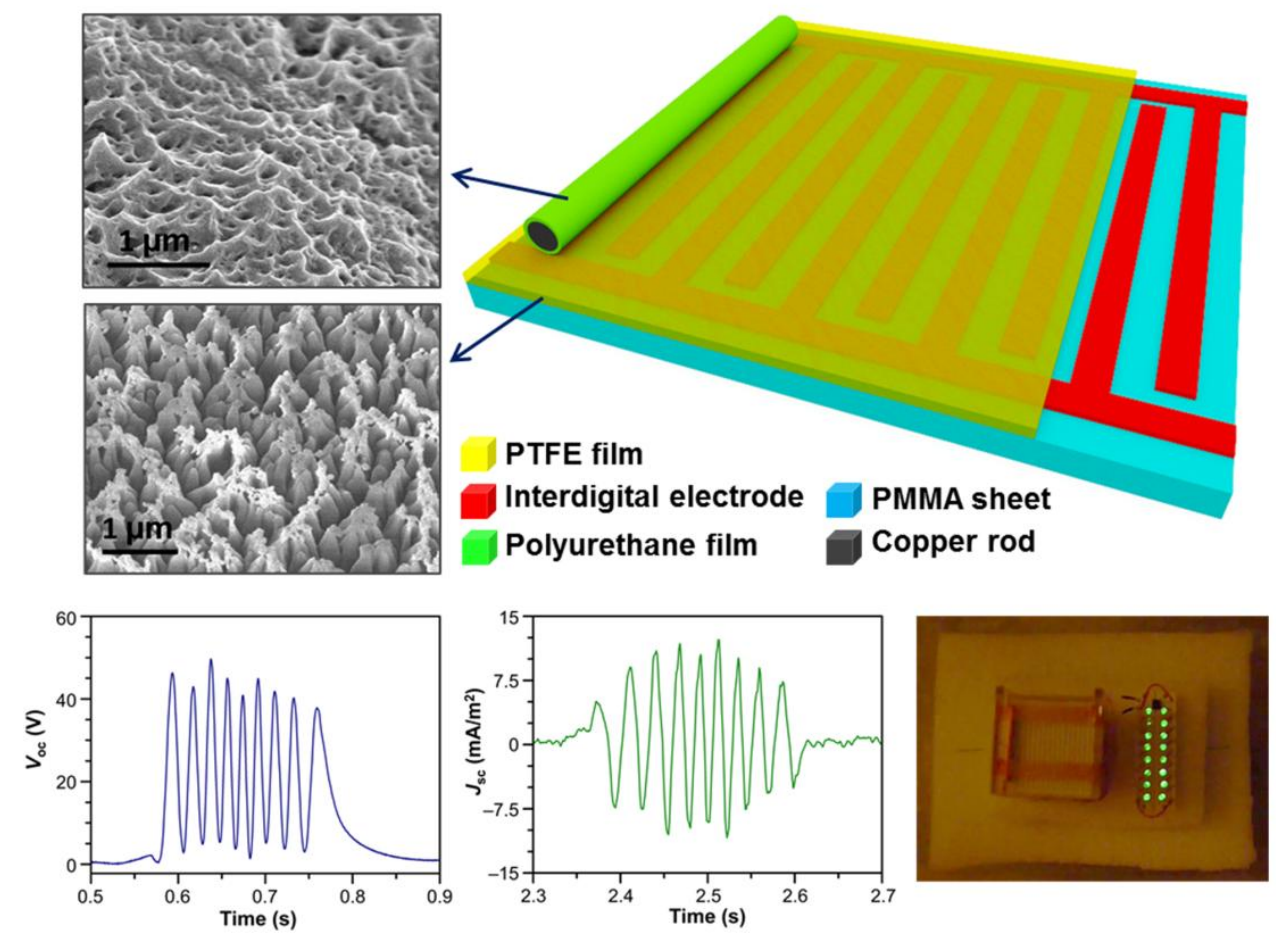

Hydropower is the most important and wildly-used renewable energy source in the environment. In this paper, a new concept combining interdigitative electrodes and multi-layered triboelectric nanogenerator has been proposed and shows the potential to harvest the ocean wave and raindrop energy. 